\title{
A novel frameshift mutation in the FERMT1 gene in a Chinese patient with Kindler syndrome
}

\author{
LI MENG $^{1 *}$, XIAOQIN YANG ${ }^{2 *}$, YUHAO WU $^{2}$, ZIJUN ZHAO $^{2}$, LIANJUAN YANG $^{1}$, \\ MING $\mathrm{LI}^{3}$, XIULI WANG ${ }^{2}$ and GUOLONG ZHANG ${ }^{2}$
}

${ }^{1}$ Department of Dermatological Mycology; ${ }^{2}$ Institute of Photomedicine, Shanghai Skin Disease Hospital, Tongji University School of Medicine, Shanghai 200443; ${ }^{3}$ Department of Dermatology, Xinhua Hospital, Shanghai Jiaotong University School of Medicine, Shanghai 200092, P.R. China

Received January 16, 2020; Accepted July 17, 2020

DOI: $10.3892 / \mathrm{etm} .2020 .9233$

\begin{abstract}
Kindler syndrome (KS) is a rare subtype of epidermolysis bullosa that is inherited in an autosomal recessive manner with mutations in FERMT1. A number of mutations in FERMT1 have been identified in KS. The current study reported a 33-year-old Chinese man who exhibited a wide variety of clinical features, including formation of blisters, photosensitivity, cutaneous atrophy and poikiloderma, telangiectasia of the face and neck, contracture of the end limbs, nail dystrophy, muscle, eye and oral damage, tympanitis, esophagus narrowing, pneumothorax and palmoplantar keratoderma. The patient's parents were healthy and the patient had no siblings or children. Peripheral blood was obtained from the patient, his parents and 100 controls, who were admitted to the Dermatology Clinic of Shanghai Skin Disease Hospital, Shanghai, China. A multi-gene panel test consisting of 541 genetic loci of monogenic hereditary diseases was performed. The results identified one novel homogenous mutation in the patient: c.1885_1901del (p.Val629fs) on exon 15 in FERMT1. The patient's parents exhibited heterogeneous identical mutations. This mutation was absent in the control group. The results of the multi-gene panel test were further verified by Sanger sequencing. Based on the clinical manifestations and genetic analysis, KS was diagnosed in the patient. The current study reported a Chinese case of KS with one novel mutation c.1885_1901del in FERMT1 and presented a brief summary of all pathogenic mutations in FERMT1 that have been reported in KS between 1984 and May 2020 via a PubMed literature search.
\end{abstract}

Correspondence to: Professor Guolong Zhang, Institute of Photomedicine, Shanghai Skin Disease Hospital, Tongji University School of Medicine, 1278 Baode Road, Shanghai 200443, P.R. China E-mail: zglamu@163.com

${ }^{*}$ Contributed equally

Key words: Kindler syndrome, fermitin family member 1, mutation, inherited epidermolysis bullosa, Chinese

\section{Introduction}

Kindler syndrome [KS; Online Mendelian Inheritance in Man (OMIM) no. 173650] is a rare subtype of inherited epidermolysis bullosa (1). Since KS was first described by Theresa Kindler in 1954 (2) and, to date, 250 affected individuals have been reported worldwide (1). KS is characterized by skin fragility acral blister formation beginning at birth or in early infancy, diffuse cutaneous atrophy, poikiloderma, photosensitivity (which is severe during childhood and usually weakens after adolescence), palmoplantar hyperkeratosis and pseudo syndactyly (1). Mucosal manifestations are also common and include hemorrhagic gingivitis and mucositis, premature loss of teeth, periodontal disease and labial leukokeratosis (1). Other mucosal findings may include ectropion, anal stenosis, esophageal strictures/stenosis, urethral stenosis/strictures, colitis and severe phimosis (1). Severe long-term complications of KS have been indicated to include mucosal strictures, periodontitis and aggressive squamous cell carcinomas (1). The phenotypic spectrum ranges from mild to severe based on the severity of symptoms, the organs involved and the age of onset (1). KS is inherited in an autosomal recessive manner and FERMT1 has been identified to be a pathogenic gene (1). To date, $>73$ unique pathogenic variants in FERMT1 have been reported in KS worldwide (1). The majority of the pathogenic variants are nonsense mutations and result in the loss of function of kindlin-1 gene (1). The diagnosis of KS is established in patients with typical clinical manifestations and is based on the identification of either biallelic FERMTI pathogenic variants on molecular genetic testing or suggestive histologic findings and/or immunolabeling on skin biopsy (1). The current study reported a Chinese patient with KS based on typical clinical manifestations and a novel FERMTI mutation, and presented a brief summary of all pathogenic mutations in FERMT1 reported in KS between 1984 and May 2020.

\section{Materials and methods}

Ethics statement and clinical participants. The current study was approved by the Ethics Committee of Shanghai Skin Disease Hospital, and was performed according to the principles of the Declaration of Helsinki (3). Peripheral blood was 
collected from the patient, his parents and 100 controls who were admitted to the Dermatology Clinic of Shanghai Skin Disease Hospital, Shanghai, China. The clinical information of all participants is presented in Table I. Written informed consent was obtained from all participants.

DNA extraction. DNA was extracted from peripheral blood using a Blood Genomic DNA Miniprep kit (cat. no. AP-MN-BL-GDNA-50; Axygen; Corning Life Sciences), according to the manufacturer's protocol.

Multi-gene panel test. Genomic DNA was extracted from the patient as aforementioned and a DNA library was constructed using a Kapa Illumina HTP library kit (cat. no. 7138008001; Roche Diagnostics) according to the manufacturer's protocol (Roche NimbleGen, Inc.). A total of $2 \mu 1$ of the DNA library was extracted and quantified using a Qubit 3.0 fluorescence quantitative analyzer (DNA library yield: $38 \mathrm{ng} / \mu \mathrm{l} \times 50 \mu \mathrm{l}$ ). Based on the quantitative fluorescence results, $100 \mathrm{ng}$ DNA library was used for electrophoresis detection with $2 \%$ agarose gel, and a library fragment at $\sim 300 \mathrm{bp}$ was considered qualified for subsequent hybridization. The DNA library was hybridized using a probe labeled with biotin $\left(47^{\circ} \mathrm{C}\right.$ for $\left.16-20 \mathrm{~h}\right)$ and the target area was captured with magnetic beads coated with streptavidin following the protocol of Roche NimbleGen, Inc. 166 ng DNA library was extracted, and a genetic skin disease gene detection panel (designed by the current study) was used to capture the target area according to the protocol of Roche NimbleGen, Inc. The panel designed in the present study included 541 genes of monogenic hereditary diseases, which comprised the following 21 bullous disease-associated genes: KRT5, KRT14, PLEC, DST, KLHL24, TGM5, DSP, PKP1, JUP, EXPH5, COL7A1, LAMA3, LAMB3, LAMC2, ITGA6, ITGB4, COL17A1, CD151, ITGA3, PLCG2 and FERMT1.

A total of $2 \mu \mathrm{l}$ of the DNA library was extracted and quantified using a Qubit 3.0 fluorescence quantifier (capture library output, $9.2 \mathrm{ng} / \mu \mathrm{l} \times 20 \mu \mathrm{l})$. According to the quantitative fluorescence results, $1 \mathrm{ng}$ of the captured library was used to detect the molecular size of the library using an Agilent 2100 Bioanalyzer (Agilent Technologies, Inc.), and a library fragment of $\sim 300$ bp was considered qualified for subsequent high-throughput sequencing. An Illumina HiSeq $\mathrm{X}$ Ten sequencer (Illumina, Inc.) was used for high-throughput sequencing of $\sim 1 \mathrm{~Gb} / \mathrm{sample}$ based on the dilution capture library of the Qubit 3.0 fluorescence quantifier and the Agilent 2100 Bioanalyzer. The sequencing reaction was performed using the paired end direction and a $2 \times 150 \mathrm{bp}$ length. PCR details are presented in Table SI.

Data analysis and interpretation. The original data files were converted from bcl to fastq format using Illumina CASAVA1.8 (Illumina, Inc.) and reads were compared to the GRCh38/hg38 human genome reference using SAMtools (v1.10.2; https://sourceforge.net/projects/samtools/), Burrows-Wheeler Aligner (v0.7.17; https://sourceforge. net/projects/bio-bwa/), Genome Analysis Toolkit (v4.1.4.1; https://github.com/broadinstitute/gatk/releases) and Picard (v1.119; https://sourceforge.net/projects/picard/) to remove repeated sequences and identify genetic variants. Nonsynonymous genetic variants in the exon region were identified. Frequencies of the identified genetic variants were required to be equal to 0 or $<1 \%$ in the Asian population in all used databases including 1,000 genomes databases (internationalgenome.org/data-portal/sample) and ExAC (exac.broadinstitute.org/dbsnp). All identified variants were evaluated by browsing through databases using gene name or disease name as search terms, including Online Mendelian Inheritance in Man (omim.org/), National Center for Biotechnology Information (NCBS) Single Nucleotide Polymorphisms database (ncbi.nlm.nih.gov/SNP/), NCBI ClinVar (ncbi.nlm.nih.gov/clinvar/) and Human Gene Mutation Database (http://www.hgmd.cf.ac.uk/ac/index. php). The putative effects on the proteins of the identified variants were explored using various prediction algorithms, including PolyPhen2 (http://genetics.bwh.harvard.edu/pph2), MutationTaster (mutationtaster.org/) and Sorting Intolerant From Tolerant (sift.bii.a-star.edu.sg). The pathogenicity of the identified variants was evaluated according to criteria established by the American College of Medial Genetics and Genomics (ACMG) (4).

Sanger sequencing. To verify the accuracy of the multi-gene panel test, direct Sanger sequencing (5) was performed to confirm whether the variants co-segregated with the disease phenotype in the patient, his parents and the control group using an ABI PRISM 3730XL automated sequencer (Applied Biosystems; Thermo Fisher Scientific, Inc.). The reverse and forward primers were as follows: 5'-AGCGGTGAATGTATG TTGTC-3' and 5'-TCCTTGTCAGAGTTTAAGCTTCT-3'.

Literature review. The term 'Kindler syndrome' was used to search the literature using PubMed between 1984 and May 2020 (https://pubmed.ncbi.nlm.nih.gov/) and the full texts of retrieved literatures were read carefully. Pathogenic mutations in FERMT1 identified in KS were summarized in Table SII.

\section{Results}

Clinical features of the patient. The patient was a 33-year-old man born to healthy non-consanguineous Chinese parents and had no siblings or children. The patient's skin was fragile and blistered easily following injury or friction at birth mainly in the knee, elbow, heel and hip that undergo high friction. Furthermore, the patient's skin gradually developed a parchment-like appearance, telangiectasia was present on the face and neck, and diffuse cutaneous atrophy and poikiloderma was present across the whole body (Fig. 1). The patient exhibited photosensitivity in childhood, which eased followed puberty. The patient's fingernails and toenails began to exhibit damage when at $\sim 7$ years old and gradually developed contracture, nail dystrophy, muscular dystrophy and muscle weakness (Fig. 1). The patient underwent pneumothorax at 22 years old and recovered following treatment. The patient also exhibited significant eye damage including nearsightedness (left eye, 500 degrees; right eye, 600 degrees), astigmatism, proneness to eye fatigue and frequent keratitis and corneal ulcers. Furthermore, the patient exhibited oral damage, including oral ulcers, gingivitis, periodontal disease, persistent gingival bleeding, prominent cavities in the right teeth and significantly decreased chewing 
Table I. Clinical information of all participants.

A, Patients

\begin{tabular}{lclll}
\hline & Age, year & Sex & Location & Date of recruitment \\
\hline Proband & 33 & Male & Shanghai, China & July, 2019 \\
Father & 60 & Male & Shanghai, China & July, 2019 \\
Mother & 58 & Female & Shanghai, China & July, 2019 \\
\hline
\end{tabular}

$\mathrm{B}$, Controls ${ }^{\mathrm{a}}$

\begin{tabular}{lcccc}
\hline Number, $\mathrm{n}$ & Age, years (mean) & Sex, Male:Female & Location & Date of recruitment \\
\hline 100 & $22-58(36)$ & $47: 53$ & Shanghai, China & October 2019-July 2020
\end{tabular}

${ }^{a}$ Inclusion criteria: Without systemic, autoimmune or genetic disease in the family.
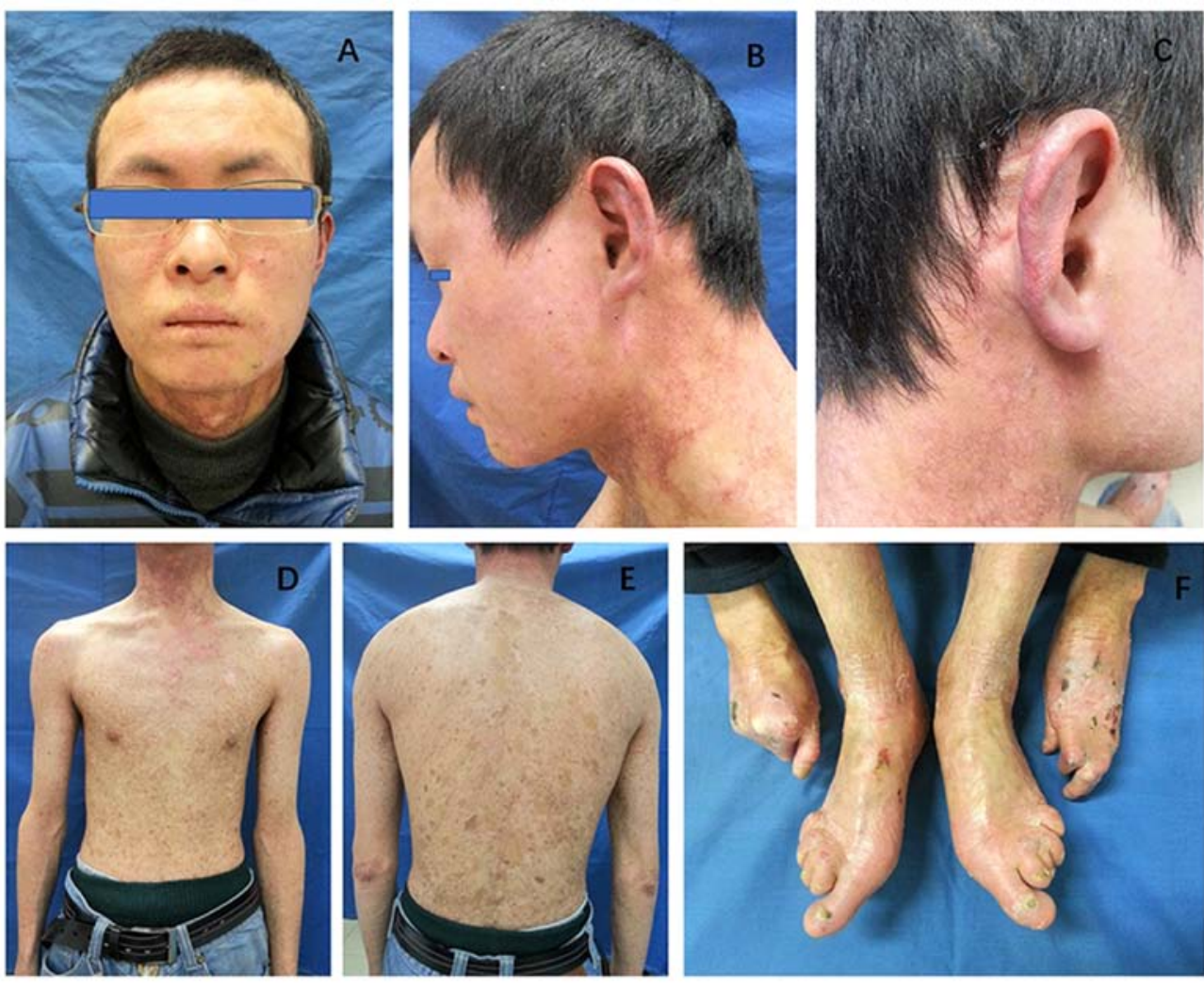

Figure 1. (A-E) Cutaneous atrophy, telangiectasia and poikiloderma in the face, neck and torso. (F) Nail dystrophy and contracture of the end of limbs.

function. The patient was diagnosed with tympanitis 2 years ago, which improved following treatment. Additionally, the patient exhibited dysphagia and a narrow esophagus, and could not swallow pills. Intestinal obstruction occurred when the patient was young; but following treatment, normal function was restored. The patient's foreskin had been removed when young. Additionally, keratinizing plaques occurred repeatedly on his palmar metatarsals. These plaques would fall off by themselves and caused the patient apparent pain following shedding. At the end of the study period, the condition of the patient was stable and the patient was able to nurse himself.
Mutation analyses. The multi-gene panel test revealed a homogeneous mutation in FERMT1, c.1885_1901del (p.Val629fs), on exon 15 in the patient. An identical, heterogeneous mutation was reported in the patient's parents, which was further confirmed by Sanger sequencing. Furthermore, this mutation was absent in the 100 controls analyzed by Sanger sequencing (Fig. 2). Combined with the clinical features and genetic analysis, the patient was diagnosed with KS. Furthermore, a heterogeneous mutation c.745C >T (p.R249X) on exon 7 in the serpin family B member 7 (SERPINB7) gene was identified in the patient using the multi-gene panel test. 


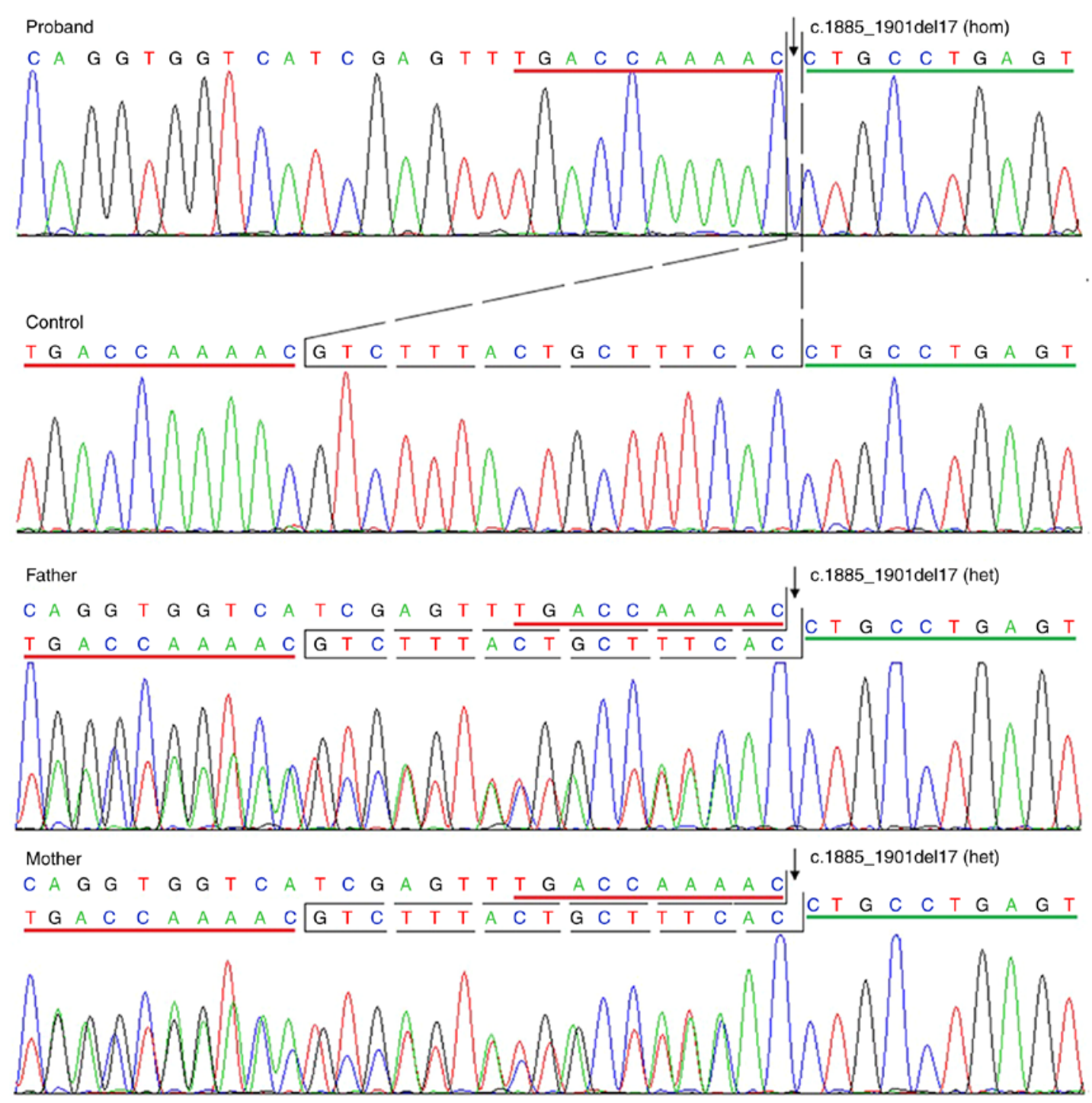

Figure 2. Sanger sequencing revealed a homogeneous mutation [c.1885_1901del (p.Val629fs)] on exon 5 in in fermitin family member 1 in the patient. The same heterogeneous mutation was reported in his parents. Furthermore, this mutation was absent in the randomly selected control group.

Review of 83 FERMT1 mutations reported in KS. Literature review from 1984 up to May 2020, and inclusive of the data in the current study, revealed $\sim 83$ different mutations in FERMTI in different populations and included frameshift (insertions and deletions), nonsense, missense and splice mutations. Mutations were located in exons 1-15, introns 1, 7-11, 13-14 and regulatory regions. Only 23 were identified to be recurrent in $>1$ report (Table SII). The results demonstrated that $>50 \%$ of the 83 mutations produced a premature termination codon and may lead to absent kindlin-1 protein or production of nonfunctional proteins.

\section{Discussion}

KS is caused by mutations in FERMT1 on chromosomal locus 20 p12.3 (1). FERMT1 contains 15 exons and spans $48.5 \mathrm{~kb}$ of genomic DNA and encodes the 677-amino acid protein kindlin, a member of the protein family of kindlin, which is an essential integrin activator and component of keratinocyte focal adhesions, and serves a regulatory role in keratinocyte migration, adhesion and proliferation (6). These features of KS may depend on the functions of kindlin-1. Numerous unique pathogenic variants in FERMT1 have been reported in KS (1). The current study reported a case of KS and identified a novel mutation in FERMT1. Additionally, a brief summary of pathogenic mutations in FERMT1 was presented.

The patient exhibited a wide range of clinical feature including fragile skin, blister formation, photosensitivity, diffuse cutaneous atrophy and poikiloderma, telangiectasia of the face and neck, contracture of the ends of limbs, nail dystrophy, muscle, eye and oral damage, tympanitis, dysphagia, a narrow esophagus, pneumothorax and palmoplantar keratoderma.Furthermore, apart from pneumothorax and tympanites, other aforementioned patient symptoms have been reported by previous studies. Additionally, numerous symptoms that have been previously reported in patients with $\mathrm{KS}$ were absent in the patient outlined in the current study, including web formation, pseudo syndactyly, genital malformation, osteoporosis (7), brachydactyly, bladder cancer (8), squamous carcinoma hyperhidrosis (9), loss of teeth, ichthyosis, microstomia cheilitis, halitosis (10), ectropion, urethral stenosis, constipation, anal atresia (11), xerosis, hypertelorism (12), ulcerative colitis, 
cholangitis, cirrhosis (13), alopecia areata (14), depression and thyroid adenoma (15). However, it remains unclear whether some of these symptoms were a result of KS or were coincidental. According to the literature search of the present study, hundreds of cases of KS have been reported. These cases have been demonstrated to exhibit similar and different symptoms, and different disease-causing mutations without an obvious genotype-phenotype correlation.

Genetic analyses revealed a homogeneous mutation in the patient: c.1885_1901del (p.Val629fs) on exon 15. Furthermore, a heterogeneous identical mutation was reported in the patient's parents. To the best of our knowledge, this mutation has not been previously reported. This mutation was determined to be pathogenic according to the ACMG guidelines (4). Based on the clinical manifestation and genetic test results, the patient was diagnosed with $\mathrm{KS}$. The mutation c.1885_1901del, which is a 17 bp deletion, generated a frameshift change of the nucleotide sequence and a premature termination codon 28 codons downstream. The resulting reading frame coded for a mutant polypeptide lacking 16 amino acids at the $\mathrm{C}$ terminus. This protein region homologous to the talin band 4.1/ezrin/radixin/moesin domain and its elimination is predicted to lead to the loss of kindlin-1 linkage function between actin cytoskeleton and integrin-associated signal platforms (10). Loss of kindlin-1 functions leads to the destruction of epithelial barriers and the inflammatory intestinal phenotype characteristic of KS (16). The current study did not perform a mutant protein expression analysis as no further samples of the patient's skin were available.

To date, c.1885_1901del (p.Val629fs) is the 5th reported mutation in Chinese patients with KS, with the other four mutations including three deletion mutations: c.994_995delCA (17), a 17252-bp deletion mutation (18) and g.63601_66617del (14), and one missense mutation, c.1343T >A (p.Met448Lys) (19). c.994_995delCA was the only mutation that was recurrent and was also reported in the Iranian population (20). Additionally, one heterogeneous mutation c.745C $>\mathrm{T}$ (p.R249X) on exon 7 in the SERPINB7 gene was identified in the patient in the current study by the multi-gene panel test. Mutations in SERPINB7 have been reported to cause Nagashima-type palmoplantar keratosis (NPPK; OMIM no. 615598), which is an autosomal recessive palmoplantar keratoderma (21). However, the c.745C $>$ T (p.R249X) mutation was heterogeneous in this patient. Therefore, the mutation did not cause NPPK. Therefore, although the patient exhibited palmoplantar keratoderma, this symptom was caused by KS, not NPKK.

Inclusive to the data of the current study, $\sim 83$ different pathogenic mutations in FERMT1 have been reported from 1984 up to May 2020. These mutations were scattered throughout the FERMT1 gene, including exons 1-15, introns 1, 7-11, 13-14 and regulatory regions exhibiting no obvious hotspots or clustering. Numerous mutations were nonsense mutations or frameshift variants (insertion and deletion variants). The majority of these mutations were reported to be homogenous in patients and few were compound heterogeneous mutations. Most of these mutations result in loss of function and may lead to absent kindlin-1 protein or the production of dysfunctional proteins (1). Only 23 mutations were revealed to be recurrent in $>1$ report. Notably, c.676insC was the most common mutation reported in 11 different reports (7,22-31) in multiple populations, including German, Albanian, Kosovian, Turkish, Serbian-Greek, Pakistani, Serbian, Australian, Indian, Brazilian and Greek Caucasian, indicating that c.676ins $\mathrm{C}$ may be a mutation hotspot and serve as a founder effector in the genetic pathogenesis of KS. Furthermore, c. $910 \mathrm{G}>\mathrm{T}$ occurred in 6 reports $(20,23,25,27,32,33)$, c. $328 \mathrm{C}>\mathrm{T}$ in 5 reports $(9,20,23,34,35)$, c. $1718+2 \mathrm{~T}>\mathrm{C}$ in 4 reports $(20,22,36,37)$ in multiple different populations and appeared to be relatively common in FERMT1. Certain mutations occurred in the same population in different reports, indicating population-specific pathogenic mutations. For instance, c.1089delG (38-40) was reported three times in only Japanese patients and c.1848_1851dupGGAA $(9,10,41)$ was reported three times in only Turkish patients. Further genotype-phenotype correlation studies are required to clarify these associations.

In conclusion, the current study reported a case of $\mathrm{KS}$ in a Chinese patient, identified one novel mutation c.1885_1901del (p.Val629fs) on exon 15 in FERMT1, and presented a summary of pathogenic FERMT1 mutations in KS. These results expand the spectrum of known FERMT1 mutations and provided a detailed mutation repertoire of FERMT1 in KS. Early and accurate diagnosis of $\mathrm{KS}$ is pivotal for the evaluation of potential complications and genetic counselling.

\section{Acknowledgements}

Not applicable.

\section{Funding}

The current work was supported by Key Projects of Shanghai Municipal Commission of Health and Family Planning (grant no. 201640016).

\section{Availability of data and materials}

The datasets generated and/or analyzed during the current study are available in the sequence read archive (SRA), https://submit.ncbi.nlm.nih.gov/subs/bioproject/, SRA access no.: PRJNA641496.

\section{Authors' contributions}

LM has been involved in drafting the manuscript and revising it critically for important intellectual content, and the acquisition of data, analysis and interpretation of data. XY, YW, $\mathrm{ZZ}$ and LY have been involved in the acquisition of data, analysis and interpretation of data. GZ, ML and XW have made substantial contributions in the conception and design of the current study. All authors read and approved the final manuscript.

\section{Ethics approval and consent to participate}

The current study was approved by the Ethics Committee of the Shanghai Skin Disease Hospital, Shanghai, China and the patient, his parents and all 100 controls provided written informed consent. 


\section{Patient consent for publication}

The patient provided written informed consent for the publication of any associated data and accompanying images.

\section{Competing interests}

The authors declare that they have no conflicting interests.

\section{References}

1. Youssefian L, Vahidnezhad H and Uitto J: Kindler Syndrome In: GeneReviews((R)). Adam MP and Pagon RA (eds), Seattle, WA, pp1-19, 2016.

2. Kindler T: Congenital poikiloderma with traumatic bulla formation and progressive cutaneous atrophy. $\mathrm{Br} \mathrm{J}$ Dermatol 66 104-111, 1954.

3. Rickham PP: Human experimentation. Code of ethics of the world medical association. Declaration of helsinki. Br Med J 2: $177,1964$.

4. Richards S, Aziz N, Bale S, Bick D, Das S, Gastier-Foster J, Grody WW, Hegde M, Lyon E, Spector E, et al: Standards and guidelines for the interpretation of sequence variants: A joint consensus recommendation of the American college of medical genetics and genomics and the association for molecular pathology. Genet Med 17: 405-424, 2015.

5. Estrada-Rivadeneyra D: Sanger sequencing. FEBS J 284: 4174, 2017.

6. Jobard F, Bouadjar B, Caux F, Hadj-Rabia S, Has C, Matsuda F, Weissenbach J, Lathrop M, Prud'homme JF and Fischer J: Identification of mutations in a new gene encoding a FERM family protein with a pleckstrin homology domain in kindler syndrome. Hum Mol Genet 12: 925-935, 2003.

7. Mansur AT, Elcioglu NH, Aydingöz IE, Akkaya AD, Serdar ZA, Herz C, Bruckner-Tuderman L and Has C: Novel and recurrent KIND1 mutations in two patients with kindler syndrome and severe mucosal involvement. Acta Derm Venereol 87: 563-565, 2007.

8. Fuchs-Telem D, Nousbeck J, Singer A, McGrath JA, Sarig O and Sprecher E: New intragenic and promoter region deletion mutations in FERMT1 underscore genetic homogeneity in kindler syndrome. Clin Exp Dermatol 39: 361-367, 2014.

9. Lai-Cheong JE, Tanaka A, Hawche G, Emanuel P, Maari C, Taskesen M, Akdeniz S, Liu L and McGrath JA: Kindler syndrome: A focal adhesion genodermatosis. Br J Dermatol 160 233-242, 2009

10. Kacar N, Semerci N, Ergin S, Pascucci M, Zambruno G and Castiglia D: A novel frameshift mutation in the KIND1 gene in Turkish siblings with kindler syndrome. Br J Dermatol 158: $1375-1377,2008$.

11. Has C, Yordanova I, Balabanova M, Kazandjieva J, Herz C, Kohlhase J and Bruckner-Tuderman L: A novel large FERMT1 (KIND1) gene deletion in kindler syndrome. J Dermatol Sci 52 209-212, 2008

12. Kartal D, Borlu M, Has C and Fölster-Holst R: A novel mutation in the fermt 1 gene in Turkish siblings with kindler syndrome. J Eur Acad Dermatol Venereol 30: 1233-1235, 2016.

13. Roda Â, Travassos AR, Soares-de-Almeida L and Has C: Kindler syndrome in a patient with colitis and primary sclerosing cholangitis: Coincidence or association? Dermatol Online J 24: 2018

14. Zhou C, Song S and Zhang J: A novel 3017-bp deletion mutation in the FERMT1 (KIND1) gene in a Chinese family with kindler syndrome. Br J Dermatol 160: 1119-1122, 2009.

15. Fukushi R, Tsuboi R, Maeda T, Kanda Y, Sakai N, Suzuki S and Harada K: A case of kindler syndrome in a young Indian female with exon deletion. Int J Dermatol 58: e19-e21, 2019.

16. Herz C, Aumailley M, Schulte C, Schlotzer-Schrehardt U, Bruckner-Tuderman L and Has C: Kindlin-1 is a phosphoprotein involved in regulation of polarity, proliferation, and motility of epidermal keratinocytes. J Biol Chem 281: 36082-36090, 2006.

17. Oh SJ, Kim SE, Lee SE and Kim SC: Homozygous deletion mutation of the fermtl gene in a Chinese patient with kindler syndrome. Ann Dermatol 28: 503-505, 2016.

18. Gao Y, Bai JL, Liu XY, Qu YJ, Cao YY, Wang JC, Jin YW, Wang $\mathrm{H}$ and Song F: A novel large deletion mutation of FERMT1 gene in a Chinese patient with Kindler syndrome. J Zhejiang Univ Sci B 16: 957-962, 2015.
19. Zheng BW, Zhu XZ, Lan Y, Ma JC and Li XQ: Unique variants in the FLG gene and FERMT1 gene in a chinese patient with ichthyosis and kindler syndrome. JAAD Case Rep 5: 1061-1064, 2019.

20. Youssefian L, Vahidnezhad H, Barzegar M, Li Q, Sotoudeh S, Yazdanfar A, Ehsani AH, Kajbafzadeh AM, Mozafari N, Daryani NE, et al: The kindler syndrome: A spectrum of FERMT1 mutations in Iranian families. J Invest Dermatol 135: 1447-1450, 2015

21. Kubo A: Nagashima-type palmoplantar keratosis: A common Asian type caused by SERPINB7 protease inhibitor deficiency. J Invest Dermatol 134: 2076-2079, 2014.

22. Techanukul T, Sethuraman G, Zlotogorski A, Horev L, Macarov M, Trainer A, Fong K, Lens M, Medenica L, Ramesh V, et al: Novel and recurrent FERMT1 gene mutations in kindler syndrome. Acta Derm-Venereol 91: 267-270, 2011.

23. Has C, Herz C, Zimina E, Qu HY, He Y, Zhang ZG, Wen TT, Gache Y, Aumailley M and Bruckner-Tuderman L: Kindlin-1 is required for RhoGTPase-mediated lamellipodia formation in keratinocytes. Am J Pathol 175: 1442-1452, 2009.

24. Kiritsi D, He Y, Pasmooij AM, Onder M, Happle R, Jonkman MF, Bruckner-Tuderman L and Has C: Revertant mosaicism in a human skin fragility disorder results from slipped mispairing and mitotic recombination. J Clin Invest 122: 1742-1746, 2012

25. Kern JS, Herz C, Haan E, Moore D, Nottelmann S, von Lilien T, Greiner P, Schmitt-Graeff A, Opitz OG, Bruckner-Tuderman L and Has C: Chronic colitis due to an epithelial barrier defect: The role of kindlin-1 isoforms. J Pathol 213: 462-470, 2007.

26. Lai-Cheong JE, Moss C, Parsons M, Almaani N and McGrath JA: Revertant mosaicism in kindler syndrome. J Invest Dermatol 132: 730-732, 2012.

27. Ashton GH, McLean WH, South AP, Oyama N, Smith FJ, Al-Suwaid R, Al-Ismaily A, Atherton DJ, Harwood CA, Leigh IM, et al: Recurrent mutations in kindlin-1, a novel keratinocyte focal contact protein, in the autosomal recessive skin fragility and photosensitivity disorder, kindler syndrome. J Invest Dermatol 122: 78-83, 2004

28. Martignago BC, Lai-Cheong JE, Liu L, McGrath JA and Cestari TF: Recurrent KIND1 (C20orf42) gene mutation, c.676insC, in a Brazilian pedigree with kindler syndrome. Br J Dermatol 157: 1281-1284, 2007.

29. Shaiq PA, Klausegger A, Muzaffar F, Bauer JW, Khan MI, Khanum A, Qamar R and Raja GK: Founder mutation c.676insC in three unrelated kindler syndrome families belonging to a particular clan from Pakistan. J Dermatol 39: 640-641, 2012.

30. Thomson MA, Ashton GH, McGrath JA, Eady RA and Moss C: Retrospective diagnosis of kindler syndrome in a 37-year-old man. Clin Exp Dermatol 31: 45-47, 2006.

31. Gkaitatzi M, Kalloniati E, Has C, Kiritsi D, Spiliopoulos T and Georgiou S: Kindler syndrome: A rare case report from Greece. Oxf Med Case Rep 2019: omz003, 2019.

32. Has C, Wessagowit V, Pascucci M, Baer C, Didona B, Wilhelm C, Pedicelli C, Locatelli A, Kohlhase J, Ashton GH, et al: Molecular basis of kindler syndrome in Italy: novel and recurrent Alu/Alu recombination, splice site, nonsense, and frameshift mutations in the KIND1 gene. J Invest Dermatol 126: 1776-1783, 2006.

33. Has $\mathrm{C}$ and Bruckner-Tuderman L: A novel nonsense mutation in kindler syndrome. J Invest Dermatol 122: 84-86, 2004.

34. de Almeida Jr HL, Heckler GT, Fong K, Lai-Cheong J and McGrath J: Sporadic Kindler syndrome with a novel mutation. An Bras Dermatol 88 (Suppl 1): S212-S215, 2013.

35. Has C, Burger B, Volz A, Kohlhase J, Bruckner-Tuderman L and Itin P: Mild clinical phenotype of kindler syndrome associated with late diagnosis and skin cancer. Dermatology 221: 309-312, 2010.

36. Fassihi H, Wessagowit V, Jones C, Dopping-Hepenstal P, Denyer J, Mellerio JE, Clark S and McGrath JA: Neonatal diagnosis of Kindler syndrome. J Dermatol Sci 39: 183-185, 2005.

37. Diociaiuti A, Zambruno G, Giancristoforo S, Proto V, Boldrini R, Castiglia D and El Hachem M: Acral skin atrophy in an infant: An early clue to Kindler syndrome diagnosis. J Eur Acad Dermatol Venereol 30: 1046-1049, 2016.

38. Siegel DH, Ashton GH, Penagos HG, Lee JV, Feiler HS, Wilhelmsen KC, South AP, Smith FJ, Prescott AR, Wessagowit $\mathrm{V}$, et al: Loss of kindlin-1, a human homolog of the Caenorhabditis elegans actin-extracellular-matrix linker protein UNC-112, causes kindler syndrome. Am J Human Genet 73: 174-187, 2003. 
39. Wada M, Masuda K, Tsuruta D, Tamai K, Lai-Cheong JE, McGrath JA and Katoh N: Case of kindler syndrome resulting from mutation in the FERMT1 gene. J Dermatol 39: 1057-1058, 2012.

40. Natsuga K, Nishie W, Shinkuma S, Nakamura H, Matsushima Y, Tatsuta A, Komine $M$ and Shimizu H: Expression of exon-8-skipped kindlin-1 does not compensate for defects of kindler syndrome. J Dermatol 61: 38-44, 2011.
41. Yildirim TT, Kaya FA, Taskesen M, Dündar S, Bozoglan A Tekin GG and Akdeniz S: Aggressive periodontitis associated with kindler syndrome in a large kindler syndrome pedigree. Turk J Pediatr 59: 56-61, 2017. 